Quim. Nova, Vol. 36, No. 3, 382-386, 2013

\title{
SÍNTESE, CARACTERIZAÇÃO E ATIVIDADE FOTOCATALÍTICA DE CATALISADORES NANOESTRUTURADOS DE TiO, DOPADOS COM METAIS
}

\author{
William Leonardo da Silva e Marla Azário Lansarin \\ Departamento de Engenharia Química, Universidade Federal do Rio Grande do Sul, R. Eng. Luis Englert, s/n, $90040-040$ Porto \\ Alegre - RS, Brasil \\ Celso Camilo Moro* \\ Departamento de Química Inorgânica, Universidade Federal do Rio Grande do Sul, Av. Bento Gonçalves, 9500, $91501-970$ Porto \\ Alegre - RS, Brasil \\ Recebido em 18/4/12; aceito em 9/10/12; publicado na web em 18/2/13

\begin{abstract}
SYNTHESIS, CHARACTERIZATION AND PHOTOCATALYTIC ACTIVITY OF NANOSTRUCTURED TiO ${ }_{2}$ CATALYSTS DOPED WITH METALS. Titanium dioxide nanostructured catalysts (nanotubes) doped with different metals (silver, gold, copper, palladium and zinc) were synthesized by the hydrothermal method in order to promote an increase in their photocatalytic activity under visible light. The catalysts were characterized by X-ray diffraction, diffuse reflectance spectroscopy, transmission electron microscopy and specific area and pore volume determination. The materials' photocatalytic activity was evaluated by rhodamine $\mathrm{B}$ decomposition in a glass batch reactor. Under UV radiation, only nanotubes doped with palladium were more active than the $\mathrm{TiO}_{2}$ P25, but the samples doped with silver, palladium and gold exhibited better results than the undoped samples under visible light.
\end{abstract}

Keywords: photocatalysis; $\mathrm{TiO}_{2}$ nanotubes; metal doped $\mathrm{TiO}_{2}$.

\section{INTRODUÇÃO}

Os processos oxidativos avançados (POAs) vêm atraindo interesse por apresentarem simplicidade operacional e elevada eficiência na degradação de compostos orgânicos tóxicos e persistentes, características importantes para o tratamento de efluentes industriais. ${ }^{1}$ Dentre os POAs, a fotocatálise heterogênea, processo que envolve reações redox induzidas pela radiação que ocorrem na superfície de semicondutores, vem sendo amplamente estudada. ${ }^{2-4}$

O catalisador mais empregado para o estudo e desenvolvimento de processos fotocatalíticos heterogêneos é o dióxido de titânio $\left(\mathrm{TiO}_{2}\right)$, na forma de anatase. Apesar de suas inúmeras vantagens, o $\mathrm{TiO}_{2}$ apresenta energia de band gap em torno de 3,2 eV, o que faz com que seja eficaz em radiações de até $385 \mathrm{~nm}$, inviabilizando a sua utilização sob a irradiação visível. Para aumentar seu campo de aplicação, é possível utilizá-lo na forma de nanocompostos, associado a outros semicondutores, ${ }^{5-7}$ ou dopado com metais. ${ }^{8-10}$ Também pode ser empregado na forma de nanotubos, que podem ser mais facilmente recuperados quando estão em solução. Além disso, em alguns casos, os NTTs podem ser reciclados e reutilizados em vários ciclos fotocatalíticos. ${ }^{11}$

A síntese de NTTs pode ser feita por diferentes métodos, dentre os quais se destacam o molde químico, a oxidação anódica eletroquímica e o tratamento hidrotérmico..$^{7,12-15}$ Os dopantes podem ser introduzidos através de impregnação, coprecipitação e dopagem ou processo sol-gel. A escolha da rota desempenha um papel significativo na atividade fotocatalítica, pois é possível que uma amostra dopada tenha boa absorção na região do visível, porém sem ação fotocatalítica. ${ }^{16}$

Embora a literatura apresente vários trabalhos estudando diferentes tipos de catalisadores dopados, ${ }^{17-22}$ ainda se faz necessário um estudo que mostre as relações entre as variáveis de preparação do catalisador na forma de nanotubos e a sua atividade fotocatalítica, considerando não somente o efeito do metal dopante, mas a forma de preparação do material base, no caso os nanotubos de titânio. Assim, o objetivo deste

*e-mail: celso@iq.ufrgs.br trabalho foi elevar a atividade fotocatalítica, na região do visível, de nanocompostos de dióxido de titânio através da dopagem com metais ( $\mathrm{Au}, \mathrm{Ag}, \mathrm{Zn}, \mathrm{Pd}$ e $\mathrm{Cu}$ ) e relacionar a atividade catalítica com a estrutura morfológica dos mesmos e com os tratamentos realizados após a síntese ( $\mathrm{pH}$ de lavagem e temperatura de calcinação).

\section{PARTE EXPERIMENTAL}

\section{Nanotubos de $\mathrm{TiO}_{2}$}

Os catalisadores nanoestruturados de $\mathrm{TiO}_{2}$ (NTTs) foram sintetizados via método hidrotérmico, descrito por Kasuga et al.. ${ }^{14}$ Inicialmente, $80 \mathrm{~g}$ de $\mathrm{NaOH}$ foram dissolvidos em $200 \mathrm{~mL}$ de água destilada e deionizada e mantidos sob agitação até dissociação completa do sólido, obtendo-se uma solução de concentração 10 mol L-1. Após, $6 \mathrm{~g}$ de $\mathrm{TiO}_{2}$ foram adicionados a esta solução, que foi mantida sob agitação por 60 min na faixa de temperatura de $50-60^{\circ} \mathrm{C}$, formando uma suspensão. Em seguida, esta suspensão foi transferida para um banho ultrassônico por 30 min e, após, distribuída igualmente em autoclaves de aço inoxidável revestidas com politetrafluoretileno. As autoclaves foram colocadas em uma estufa a $140{ }^{\circ} \mathrm{C}$ por $72 \mathrm{~h}$. $\mathrm{O}$ material coletado das autoclaves foi transferido para um béquer e lavado com água destilada e $\mathrm{HNO}_{3} 0,3 \mathrm{~mol} \mathrm{~L}^{-1}$ até que a solução de lavagem apresentasse o valor de $\mathrm{pH}$ desejado, o qual passou a ser denominado $\mathrm{pH}$ de síntese. Finalmente, o material foi filtrado e secado em uma estufa a $80^{\circ} \mathrm{C}$ por $15 \mathrm{~h}$.

Esses materiais foram submetidos à calcinação em diferentes temperaturas para a conversão em anatase. A denominação dos catalisadores assim obtidos foi NTT-x-y onde "x" é o pH de síntese e "y" a temperatura de calcinação.

\section{Dopagem com metais}

Foram preparados 7 catalisadores nanoestruturados dopados com metais, sendo utilizados cobre $(\mathrm{Cu})$, prata $(\mathrm{Ag})$, ouro $(\mathrm{Au})$, zinco (Zn) e paládio $(\mathrm{Pd})$. 
$\mathrm{O}$ procedimento utilizado para dopagem com $\mathrm{Cu}$ foi uma adaptação do método empregado por Han e colaboradore ${ }^{17}$ e consistiu em adicionar os nanotubos (NTT-4) em uma solução de sulfato de cobre $\left(\mathrm{CuSO}_{4} \cdot 5 \mathrm{H}_{2} \mathrm{O}\right)$ com $\mathrm{HNO}_{3} 0,3 \mathrm{~mol} \mathrm{~L}^{-1}$. Em seguida, se deixou a solução em repouso por $15 \mathrm{~h}$ para facilitar o contato entre os reagentes. Após, o material foi filtrado a vácuo, secado por $24 \mathrm{~h}$ a $80{ }^{\circ} \mathrm{C}$ e calcinado por $90 \mathrm{~min}$ a $600{ }^{\circ} \mathrm{C}$. O catalisador foi denominado NTT-4-600/Cu.

Para a dopagem com prata foram usados dois métodos de síntese. No primeiro, similar ao trabalho de Sahoo e colaboradores, ${ }^{18}$ adicionou-se $1 \mathrm{~g}$ de NTT-4 em 1,3 mL de $\mathrm{AgNO}_{3}$ 0,073 $\mathrm{mol} \mathrm{L}^{-1}$, completando com água deionizada até $5 \mathrm{~mL}$ e deixando-se a solução no escuro por $24 \mathrm{~h}$. Em seguida, a solução foi seca por cerca de $24 \mathrm{~h}$ a $80{ }^{\circ} \mathrm{C}$ e o material obtido foi calcinando a $600{ }^{\circ} \mathrm{C}$ por $90 \mathrm{~min}$. Este catalisador foi denominado NTT-4-600/Ag+escuro. O segundo procedimento se assemelha ao primeiro, está baseado no trabalho de Ko e colaboradores, ${ }^{19}$ porém a solução de nanotubos e $\mathrm{AgNO}_{3}$ foi deixada por 60 min sob agitação magnética em um reator batelada encamisado irradiado por uma lâmpada emitindo na região do ultravioleta para promover a redução da prata. Ao final, filtrou-se a vácuo, secando e calcinando nas mesmas condições do primeiro procedimento. $\mathrm{O}$ catalisador assim obtido foi denominado NTT-4-600/Ag+UV.

Dois catalisadores foram dopados com nanopartículas de ouro conforme procedimento análogo ao de Zhu e colaboradores. ${ }^{20}$ No primeiro procedimento, adicionou-se $0,750 \mathrm{~g}$ de NTT- 4 em $4 \mathrm{~mL}$ de uma solução de ouro coloidal na concentração de 5,0 x $10^{-3} \mathrm{~mol} \mathrm{~L}^{-1}$. A suspensão foi sonicada por 30 min e lavada com água destilada. O material resultante foi seco em estufa $\left(24 \mathrm{~h} \mathrm{a} 80^{\circ} \mathrm{C}\right)$, separado em duas partes sendo que uma destas foi calcinada $\left(120 \mathrm{~min}\right.$ a $\left.500{ }^{\circ} \mathrm{C}\right)$, resultando no catalisador NTT-4-500/Au ${ }^{\mathrm{I}}$. No segundo procedimento, adicionou-se $0,500 \mathrm{~g}$ de NTT-4-500 em 2,5 mL da mesma solução coloidal de ouro resultando no catalisador NTT-4-500/Au ${ }^{\mathrm{II}}$. O material foi submetido ao mesmo procedimento que o anterior até a etapa de secagem. A diferença entre os métodos é que no primeiro procedimento uma parte da amostra seca foi separada e calcinada após dopagem $\left(500{ }^{\circ} \mathrm{C}\right.$ por $\left.120 \mathrm{~min}\right)$, enquanto que no segundo não houve calcinação após a dopagem.

A síntese do catalisador dopado com $\mathrm{Zn}$ baseou-se no trabalho de Liu e colaboradores. ${ }^{21}$ Para isso, $0,5 \mathrm{~g}$ de acetato de zinco foram dissolvidos em $100 \mathrm{~mL}$ de etanol absoluto, sob agitação magnética, por 30 min e na temperatura ambiente, para formação de uma suspensão transparente. Em seguida, os nanotubos foram adicionados sob forte agitação magnética. Então, distribuiu-se a suspensão obtida em 3 autoclaves de aço inoxidável revestidas com politetrafluoretileno e colocadas em estufa por $120 \mathrm{~min}$ a $130^{\circ} \mathrm{C}$. Após, filtrou-se a vácuo e lavou-se com água destilada. Ao final, secou-se por $24 \mathrm{~h}$ a $80{ }^{\circ} \mathrm{C}$ e calcinou-se a $600{ }^{\circ} \mathrm{C}$ por $120 \mathrm{~min}$, produzindo o catalisador NTT-4-600/Zn.

$\mathrm{Na}$ dopagem com nanopartículas de paládio empregou-se a mesma metodologia que a do ouro. Adicionaram-se os nanotubos (NTT-4) em 2,5 mL de uma solução de nanopartículas de paládio na concentração de $2,5 \times 10^{-3} \mathrm{~mol} \mathrm{~L}^{-1}$. A suspensão foi sonicada por 30 min e deixada em repouso por $48 \mathrm{~h}$. O material resultante foi seco em estufa $\left(24 \mathrm{~h}\right.$ a $\left.80^{\circ} \mathrm{C}\right)$ e finalmente calcinado $\left(120 \mathrm{~min}\right.$ a $\left.500^{\circ} \mathrm{C}\right)$. O catalisador preparado foi denominado NTT-4-500/Pd.

\section{Caracterização}

A estrutura cristalina das amostras foi analisada através de difração de raios $\mathrm{X}$, medida em um difratômetro Bruker, modelo D2 Phaser, com radiação $\mathrm{CuK}_{\alpha}$. As amostras foram escaneadas na faixa de $2 \theta$ de $10^{\circ}$ a $70^{\circ}$, com uma tensão de aceleração de corrente aplicada de $30 \mathrm{kV}$ e $10 \mathrm{~mA}$, respectivamente. A área superficial foi calculada pelo método de Brunauer-Emmett-Teller (BET) em equipamento TriStar $^{\circledR}$ II 3020 da Micrometrics ${ }^{\circledR}$. A energia de band gap foi determinada utilizando-se dados de espectroscopia de refletância difusa, medidas em um espectrofotômetro UV-Visível Cary 100 Scan Spectrophotometer, Varian, com um assessório para medidas de refletância difusa, na faixa de comprimento de onda de 200-600 nm usando-se, posteriormente, a função Kubelka-Munk.

\section{Atividade fotocatalítica}

Os testes para a determinação da atividade fotocatalítica foram realizados em um reator de $50 \mathrm{~mL}$, imerso em banho termostático para manter a temperatura em $30^{\circ} \mathrm{C}$. A fonte de luz visível foi uma lâmpada de vapor de mercúrio de $125 \mathrm{~W}$ associada a um filtro de policarbonato para barrar os comprimentos de onda inferiores a 385 $\mathrm{nm}$. Para luz ultravioleta foi utilizada uma lâmpada similar, porém com o bulbo modificado. A concentração de catalisador foi de 0,7 $\mathrm{g} \mathrm{L}^{-1}$, adicionado a $25 \mathrm{~mL}$ de rodamina B $20 \mathrm{mg} \mathrm{L}^{-1}$. Optou-se por realizar os experimentos em $\mathrm{pH}$ natural da $\mathrm{RhB}(4,3)$, pois a adição de novos íons na solução, necessários para ajuste de $\mathrm{pH}$, poderia interferir nos resultados. A suspensão, inicialmente, foi mantida no escuro, sob agitação magnética e aeração por 60 min para se atingir o equilíbrio de adsorção. Em seguida, a suspensão foi exposta à radiação por $60 \mathrm{~min}$. As amostras foram coletadas com o auxílio de uma seringa e cateter e armazenadas em eppendorfs protegidos da luz. Para remoção do catalisador, as amostras foram centrifugadas por 20 min a 5000 rpm. Para determinação da concentração de rodamina B foi realizada leitura em espectrofotômetro Varian Cary 100 a 553 $\mathrm{nm}$, sendo que as amostras foram diluídas 10 vezes. Os ensaios foram realizados em duplicata.

\section{RESULTADOS E DISCUSSÃO}

\section{Energia de band gap $\left(\mathrm{E}_{\mathrm{g}}\right)$}

A Tabela 1 apresenta os valores de $\mathrm{E}_{\mathrm{g}}$ e os valores dos comprimentos de onda a partir dos quais não ocorre absorção de radiação pelo material. É possível verificar que, para um mesmo $\mathrm{pH}$ de síntese, o aumento da temperatura de calcinação foi acompanhado por uma redução da $\mathrm{E}_{\mathrm{g}}$. Nos fotocatalisadores dopados foi possível verificar que o metal dopante promove uma redução na energia de band gap e, assim, um aumento na atividade fotocatalítica, conforme esperado. ${ }^{12}$ Todos os catalisadores sintetizados, exceto os não calcinados, apresentaram um valor de $\mathrm{E}_{\mathrm{g}}$ menor ou igual ao precursor P-25 Degussa. Entretanto, a absorção de radiação em comprimentos de onda na região do visível, por si só, não assegura um aumento na atividade fotocatalítica, pois esta depende, dentre outros fatores, da taxa de recombinação das espécies fotogeradas.

\section{Área superficial e volume de poros}

A área superficial $\left(\mathrm{S}_{\mathrm{BET}}\right)$, o diâmetro e o volume de poros das amostras foram determinados por meio do método BET. Os resultados obtidos estão listados na Tabela 2 e ilustrados nas Figuras 1 e 2.

Na Tabela 2 verifica-se um aumento significativo da área específica e do volume de poros dos nanotubos (antes da dopagem e da calcinação) quando comparados com o catalisador comercial P-25 Degussa. Além disso, o aumento na temperatura de calcinação promove uma sinterização dos microporos, o que leva a uma diminuição nos volumes totais de poros, a um aumento no diâmetro médio dos mesmos e a uma diminuição da área superficial. A respeito do $\mathrm{pH}$ de síntese, Lee e colaboradores ${ }^{23}$ citam que a remoção do íon sódio na etapa de lavagem proporciona o aumento da área específica, o que pode ser observado nas amostras NTT-4 e NTT-7, ambas calcinadas a $600{ }^{\circ} \mathrm{C}$. 
Tabela 1. Energias de band gap determinadas pelos espectros de absorção UV-Visível com os respectivos comprimentos de onda

\begin{tabular}{|c|c|c|}
\hline Catalisador & $\mathrm{E}_{\mathrm{g}}(\mathrm{eV})$ & $\lambda(\mathrm{nm})$ \\
\hline P-25 Degussa & 3,34 & 371,25 \\
\hline NTT-4 & 3,41 & 363,63 \\
\hline NTT-4-500 & 3,29 & 376,89 \\
\hline NTT-4-600 & 3,19 & 388,71 \\
\hline NTT-7 & 3,49 & 355,29 \\
\hline NTT-7-500 & 3,33 & 372,36 \\
\hline NTT-7-600 & 3,15 & 393,64 \\
\hline NTT-4-600/Zn & 3,13 & 396,16 \\
\hline NTT-4-600/Cu & 3,11 & 398,70 \\
\hline NTT-4-500/Pd & 3,15 & 393,64 \\
\hline NTT-4-600/Ag+escuro & 3,16 & 392,40 \\
\hline NTT-4-600/Ag+UV & 3,18 & 389,90 \\
\hline NTT-4-500/Au ${ }^{\mathrm{I}}$ & 3,23 & 383,89 \\
\hline NTT-4-500/Au ${ }^{\mathrm{II}}$ & 3,27 & 379,20 \\
\hline
\end{tabular}

Tabela 2. Área superficial, diâmetro e volume de poros dos NTTs e fotocatalisadores dopados

\begin{tabular}{lccc}
\hline Catalisador & $\mathrm{S}_{\mathrm{BET}}\left(\mathrm{m}^{2} \mathrm{~g}^{-1}\right)$ & $\mathrm{Dp}(\mathrm{nm})$ & $\mathrm{Vp}\left(\mathrm{cm}^{3} \mathrm{~g}^{-1}\right)$ \\
\hline P-25 Degussa & 56 & 4,8 & 0,07 \\
NTT-4 & 280 & 5,3 & 0,45 \\
NTT-4-500 & 150 & 5,5 & 0,21 \\
NTT-4-600 & 101 & 6,3 & 0,18 \\
NNT-7 & 287 & 4,8 & 0,41 \\
NTT-7-500 & 90 & 5,3 & 0,15 \\
NTT-7-600 & 68 & 5,5 & 0,10 \\
NTT-4-600/Zn & 59 & 5,8 & 0,09 \\
NTT-4-600/Cu & 89 & 7,2 & 0,18 \\
NTT-4-500/Pd & 168 & 6,0 & 0,24 \\
NTT-4-600/Ag+escuro & 117 & 5,6 & 0,17 \\
NTT-4-600/Ag+UV & 84 & 5,4 & 0,12 \\
NTT-4-500/Au & & 6,5 & 0,23 \\
NTT-4-500/Au & & 6,3 & 0,17 \\
\hline
\end{tabular}

Em relação à dopagem, observa-se que as amostras dopadas com zinco (NTT-4-600/Zn), cobre (NTT-4-600/Cu), prata (um dos procedimentos, NTT-4-600/Ag+UV) e com ouro (NTT-4-500/Au ${ }^{\mathrm{I}} \mathrm{e}$ NTT-4-500/Au ${ }^{\mathrm{II}}$ ) provocam uma diminuição no valor da área específica em relação à amostra não dopada, diferente do que acontece com a amostra dopada com paládio (NTT-4-500/Pd) e outro procedimento com prata (NTT-4-600/Ag+escuro).

As Figuras 1 e 2 mostram as isotermas de adsorção e a distribuição de volume e diâmetro dos poros de alguns dos precursores obtidos. Embora todas as amostras tenham sido caracterizadas, são apresentados apenas os perfis das amostras não dopadas, como exemplo, pois como mostra a Tabela 2 , a presença do metal dopante pouco alterou as características do precursor.

As isotermas são do tipo IV com curvas de histerese H3, de acordo com a classificação de Brunauer-Deming-Deming-Teller (BDDT), e a distribuição de diâmetro de poros permite afirmar que são estruturas com mesoporos (de acordo com a União Internacional de Química Aplicada - IUPAC).

\section{Análises de DRX}

A Figura 3 apresenta as análises de difração raios $\mathrm{X}$ realizadas para as amostras do NTT-4, NTT-4-500 e NTT-4-600. Observa-se

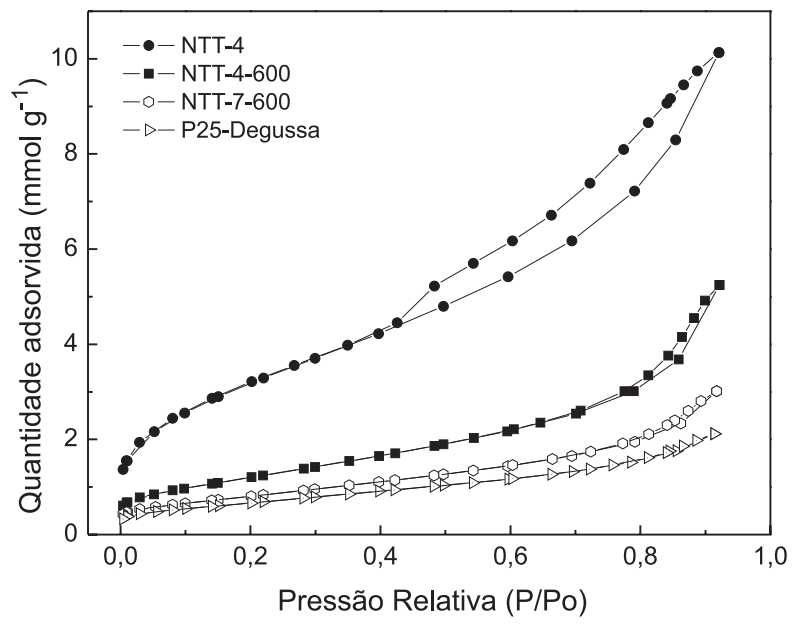

Figura 1. Isotermas de adsorção/dessorção de $N_{2}$ dos catalisadores $P-25$ Degussa, NTT-4, NTT-4-600 e NTT-7-600

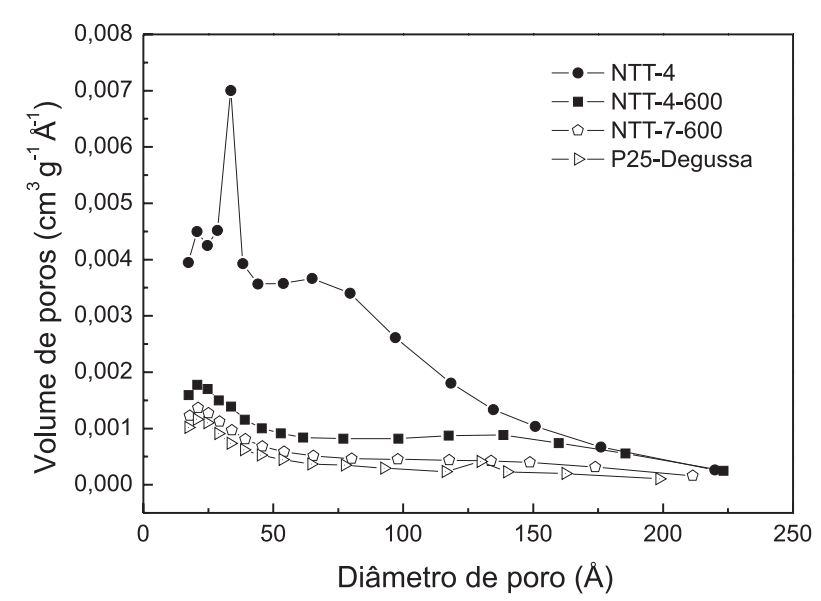

Figura 2. Distribuição de poros dos catalisadores P-25 Degussa, NTT-4, NTT-4-600 e NTT-7-600

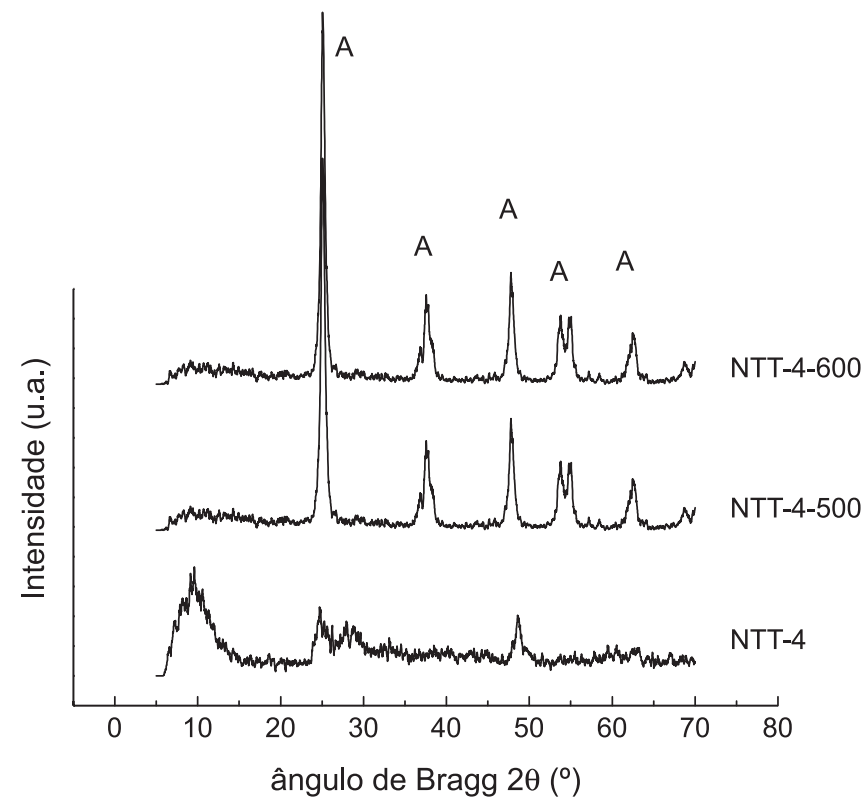

Figura 3. Difratogramas de raios X dos catalisadores NTT-4-600, NTT-4-500 e NTT-4 (A = anatase $)$ 
que o difratograma da amostra que não foi calcinada, NTT-4, é típico para material amorfo, o que prova a necessidade da calcinação para ordenação dos átomos no cristal e obtenção de uma fase cristalina. Além disso, quanto maior a temperatura de calcinação, mais definidos são os picos, o que evidencia um aumento da cristalinidade do material. Nas temperaturas estudadas não houve formação de rutilo, o que indica que, nesse caso, é necessária uma temperatura superior a $600{ }^{\circ} \mathrm{C}$ para que haja a conversão de anatase para rutilo.

$\mathrm{O}$ processo de dopagem nos nanotubos de $\mathrm{TiO}_{2}$ não promove a formação de novos picos, conforme pode ser visto na Figura 4, que apresenta difratogramas de alguns dos catalisadores dopados. Os difratogramas dos catalisadores dopados com $\mathrm{Au}, \mathrm{Pd}$ e $\mathrm{Cu}$ também não apresentaram picos característicos desses metais e optou-se por não incluí-los na figura.

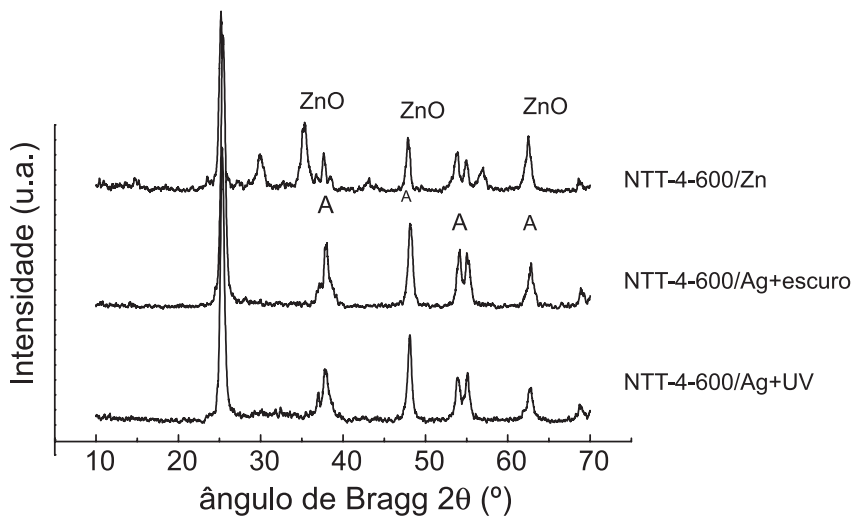

Figura 4. Difratogramas de raios $X$ dos catalisadores NTT-4-500/Zn, NTT4-600/Ag+escuro $(A=$ anatase $)$

Resultado semelhante foi obtido por Chai e colaboradores,${ }^{24}$ que avaliaram o efeito da variação da temperatura de calcinação (400 a $700{ }^{\circ} \mathrm{C}$ ) nas características de NTTs dopados com prata. Destaca-se que apenas no difratograma da amostra dopada com zinco é possível verificar pico de $\mathrm{ZnO}$ na amostra, entendendo-se que as nanopartículas estão aglomeradas.

\section{Microscopia eletrônica de transmissão (MET)}

A Figura 5 apresenta a imagem das nanopartículas de ouro depositadas sobre os NTTs antes da calcinação. Nessa figura pode-se observar a estrutura dos nanotubos com diâmetros em torno de $10 \mathrm{~nm}$, bem como a presença de nanoesferas de ouro.

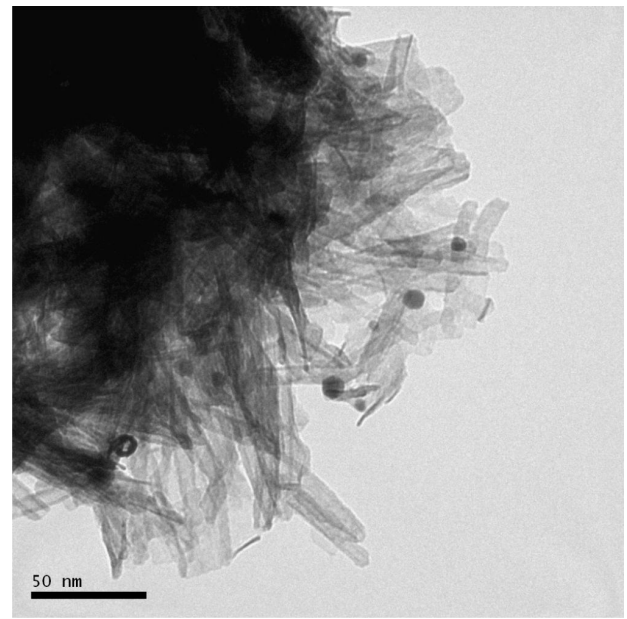

Figura 5. Imagens da MET de NTT-4 dopado com Au. Aumento $500 \mathrm{~K}$
A figura em questão exemplifica o que ocorre com os demais catalisadores dopados. Imagens apresentando nanopartículas de Au, nanotubos sintetizados, nanopartículas de Pd sobre nanotubos não calcinados e o catalisador NTT-4-500/Au ${ }^{\mathrm{I}}$ podem ser observadas nas Figuras $1 \mathrm{~S}$ a $5 \mathrm{~S}$, material suplementar.

\section{Atividade fotocatalítica}

A atividade fotocatalítica foi avaliada comparando-se a velocidade específica de reação (k) obtida na degradação de rodamina B e, também, pelo percentual total de degradação alcançado após 60 min de reação. $\mathrm{O}$ valor de $\mathrm{k}$ foi calculado assumindo-se que a reação seja de pseudoprimeira ordem, com base em estudos anteriores. ${ }^{25}$ Foram realizados ensaios preliminares para se estabelecer as concentrações de catalisador e substrato que maximizam o percentual de degradação quando a reação ocorre no aparato experimental empregado no presente trabalho.

Os ensaios de degradação sob radiação UV foram realizados nas seguintes condições: concentração do catalisador $0,70 \mathrm{~g} \mathrm{~L}^{-1}$, concentração de $\mathrm{RhB}$ de $20 \mathrm{mg} \mathrm{L}^{-1}$, temperatura de operação de $30^{\circ} \mathrm{C}, \mathrm{pH}$ 4,3 e radiação UV de $54 \mathrm{~W} \mathrm{~m}^{-2}$.

Em testes realizados na ausência de catalisador, observou-se que após 60 min de reação, sob radiação $\mathrm{UV}$, o valor de $\mathrm{C} / \mathrm{C}_{0}$ caiu para 0,86, o que indica que $14 \%$ do corante foi degradado por fotólise UV. Nos experimentos realizados sob radiação visível, a fotólise ocasionou 4\% de degradação após 60 min de reação. Os resultados que passam a ser apresentados se referem à degradação total (fotólise e fotocatálise). A Tabela 3 mostra os respectivos valores da velocidade específica de reação $(\mathrm{k})$ dos catalisadores estudados e a Figura 6 apresenta o percentual de degradação do corante após 60 min de reação sob radiação UV.

Tabela 3. Velocidades específicas de reação dos ensaios sob radiação UV e Visível*

\begin{tabular}{lccc}
\hline Catalisador & $\mathrm{k}_{\mathrm{UV}} \times 10^{3}\left(\mathrm{~min}^{-1}\right)$ & $\mathrm{k}_{\mathrm{Vis}} \times 10^{3}\left(\mathrm{~min}^{-1}\right)$ & $\mathrm{k}_{\mathrm{UV}} / \mathrm{k}_{\mathrm{Vis}}$ \\
\hline P-25 Degussa & 43,4 & 2,6 & 16,69 \\
NTT-4-500 & 30,0 & 2,8 & 10,71 \\
NTT-4-600 & 31,4 & 1,5 & 20,93 \\
NTT-4-600/Zn & 10,2 & 1,5 & 6,80 \\
NTT-4-600/Cu & 10,8 & 2,3 & 4,70 \\
NTT-4-500/Pd & 49,5 & 3,0 & 16,50 \\
NTT-4-600/Ag+escuro & 21,8 & 4,6 & 4,74 \\
NTT-4-600/Ag+UV & 10,6 & 3,3 & 3,21 \\
NTT-4-500/Au & 12,7 & 4,3 & 2,95 \\
NTT-4-500/Au $^{\mathrm{II}}$ & 9,5 & 1,5 & 6,33 \\
\hline *C $_{\text {cat }}=0,70 \mathrm{~g} \mathrm{~L}^{-1}, \mathrm{C}_{\mathrm{rbB}}=20 \mathrm{mg} \mathrm{L}^{-1}, \mathrm{~T}=30^{\circ} \mathrm{C}, \mathrm{pH}=4,3, \mathrm{I}_{\mathrm{rad} \mathrm{UV}}=54 \mathrm{~W} \mathrm{~m}^{-2} \mathrm{e}$ \\
$\mathrm{I}_{\text {rad Vis }}=120 \mathrm{~W} \mathrm{~m}^{-2}$
\end{tabular}

A amostra dopada com paládio apresentou um bom desempenho em comparação com as demais amostras dopadas. O valor encontrado de $\mathrm{k}$ foi de $49,5 \times 10^{-3} \mathrm{~min}^{-1}$, muito próximo do catalisador comercial $\mathrm{P}-25$, que é de $43,4 \times 10^{-3} \mathrm{~min}^{-1}$, e superior ao da amostra não dopada NTT-4-600 que apresenta um k de 31,4 x 10 $0^{-3} \mathrm{~min}^{-1}$, proporcionando uma degradação de $93 \%$ do corante em 60 min de ensaio fotocatalítico. As demais amostras dopadas apresentaram valores inferiores da velocidade específica de reação em relação à amostra de nanotubos não dopada e ao catalisador comercial.

Os ensaios de degradação sob radiação visível foram realizados nas mesmas condições que os ensaios sob UV, porém com intensidade de radiação de $120 \mathrm{~W} \mathrm{~m}^{-2}$. A Figura 7 apresenta o percentual de degradação do corante após 60 min de reação.

Dentre as amostras dopadas, as que apresentaram os melhores resultados foram NTT-4-500/Pd, com uma velocidade específica de 


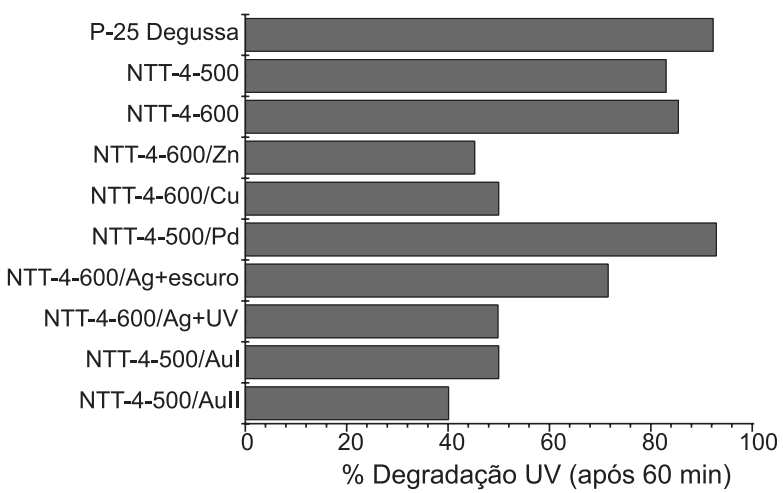

Figura 6. Percentual de degradação do corante sob a radiação UV após 60 min de reação $\left(C_{c a t}=0,70 \mathrm{~g} \mathrm{~L}^{-1}, C_{r h B}=20 \mathrm{mg} \mathrm{L}^{-1}, T=30^{\circ} \mathrm{C}, \mathrm{pH}=4,3\right.$ e $I_{\text {rad }}$ $=54 \mathrm{~W} \mathrm{~m}^{-2}$ )

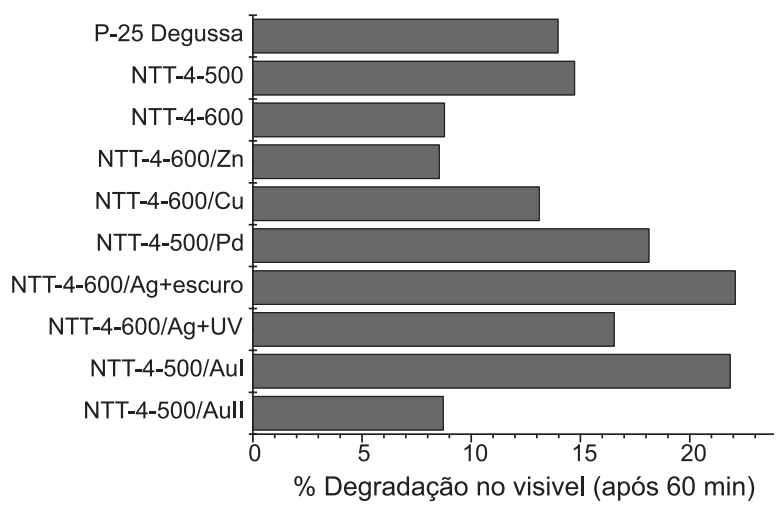

Figura 7. Percentual de degradação do corante sob a radiação visível após $60 \mathrm{~min}$ de reação $\left(C_{c a t}=0,70 \mathrm{~g} \mathrm{~L}^{-1}, C_{r h B}=20 \mathrm{mg} \mathrm{L}^{-1}, T=30^{\circ} \mathrm{C}, \mathrm{pH}=4,3 \mathrm{e}\right.$ $\left.I_{\text {rad }}=120 \mathrm{~W} \mathrm{~m}^{-2}\right)$

reação para radiação visível de 3,0 x 10-3 $\mathrm{min}^{-1}$, NTT-4-600/Ag+escuro $\left(\mathrm{k}=4,6 \times 10^{-3} \mathrm{~min}^{-1}\right)$, NTT-4-500/Au ${ }^{\mathrm{I}}\left(\mathrm{k}=4,3 \times 10^{-3} \mathrm{~min}^{-1}\right)$ e NTT4-600/Ag+UV com $\mathrm{k}=3,3 \times 10^{-3} \mathrm{~min}^{-1}$.

De maneira geral, os catalisadores são mais ativos sob radiação UV do que sob radiação visível; para o P-25, por exemplo, a relação $\mathrm{k}_{\mathrm{UV}} / \mathrm{k}_{\mathrm{VIS}}$ é 16,7 . A dopagem com prata proporcionou aumento do $\mathrm{k}_{\mathrm{VIS}}$ para ambas as amostras, de tal maneira que a relação $\mathrm{k}_{\mathrm{UV}} / \mathrm{k}_{\mathrm{VIS}}$ passa a ser de 4,74 (NTT-4-600/Ag+escuro) e 3,21 (NTT-4-600/Ag+UV). Segundo Ko e colaboradores, ${ }^{19}$ a modificação da superfície de $\mathrm{TiO}_{2}$ com partículas de $\mathrm{Ag}$ proporciona um aumento na atividade fotocatalítica devido à inibição da recombinação dos pares elétron/vacância.

\section{CONCLUSÃO}

O presente trabalho buscou relacionar a atividade fotocatalítica de nanotubos de $\mathrm{TiO}_{2}$ dopados com diferentes metais com o tratamento dado ao material após a síntese. Observou-se que: (i) uma síntese em pH levemente ácido $(4,0)$ favorece a atividade fotocatalítica; (ii) o aumento da temperatura de calcinação proporciona a formação de uma fase mais cristalina (anatase), aumentando a atividade fotocatalítica, porém com a diminuição da área superficial específica e volume de poros; e também que: (iii) a dopagem com metais leva a valores de energia de band gap menores do que os das amostras não dopadas, indicativo de que podem ser ativadas com radiação de comprimentos de onda maiores.

O catalisador comercial P-25 Degussa apresentou o melhor desempenho sob radiação UV, degradando 93\% de RhB em 60 min de reação. Sob radiação visível, entretanto, algumas amostras de nanotubos dopados apresentaram boa atividade fotocatalítica, superando o precursor P-25. Dentre estas, as que apresentaram os melhores resultados foram NTT-4-500/Pd, NTT-4-600/Ag+escuro, NTT-4-500/Au ${ }^{\mathrm{I}}$ e NTT-4-600/Ag+UV.

Os resultados obtidos no presente trabalho permitem concluir que a dopagem com metais é um dos caminhos a ser percorrido na busca de materiais que apresentem boa atividade fotocatalítica sob radiação visível.

\section{MATERIAL SUPLEMENTAR}

Figuras 1S a $5 \mathrm{~S}$ mostram fotografias obtidas com microscopia eletrônica de transmissão de nanotubos de $\mathrm{TiO}_{2}$ (NTT-4 e NTT-7) bem como nanopartículas de Au e o catalisador NTT-4-500/Au ${ }^{\mathrm{I}} \mathrm{e}$ nanopartículas de Pd depositadas sobre NTT-4. Essas figuras estão disponíveis em http://quimicanova.sbq.org.br na forma de arquivo PDF, com acesso livre.

\section{AGRADECIMENTOS}

Ao $\mathrm{CNPq}$ pelo auxílio financeiro para a realização do presente trabalho.

\section{REFERÊNCIAS}

1. Chiron, S.; Fernandes-Alba, A.; Rodriguez, A.; Garcia-Calvo, E.; Water Res. 2000, 34, 366.

2. Chen, D.; Ray, A. K.; Water Res. 1998, 32, 3223.

3. Prestes, T. H.; Gibbon, D. O.; Lansarin, M. A.; Moro, C. C.; Quim. Nova 2010, 33, 798 .

4. Nakamura, L. K. O.; Vaz, J. M.; Quim. Nova 2011, 34, 1173.

5. Yang, S. G.; Quan, X.; Li, X. Y.; Liu, Y. Z.; Chen, S.; Chen, G. H.; Chem. Phys. 2004, 6, 659.

6. Guo, Y. F.; Quan, X.; Liu, N.; Zhao, H. M.; Chen, S.; Environ. Sci. Technol. 2007, 41, 4422.

7. Chen, W.; Guo, X.; Zhang, S.; Jin, Z. ; J. Nanoparticle Res. 2007, 9, 1173.

8. Einaga, H.; Ibusuki, T.; Futamura, S.; Environ. Sci. Technol. 2004, 38, 285.

9. Kozlova, E. A.; Vorontsov, A. V.; Appl. Catal. B 2006, 63, 114.

10. You, X. F.; Chen, F.; Zhang, J. L.; Anpo, M.; Catal. Lett. 2005, 102, 247.

11. Costa, L. L; Prado, A. G. S.; J. Photochem. Photobiol. A 2009, 201, 45.

12. Bavykin, D. V.; Friedrich, J. M.; Walsh, F. C.; Adv. Mater. 2006, 18, 2807.

13. Ou, H.; Lo, S.; Sep. Purif. Technol. 2007, 58, 179.

14. Kasuga, T.; Hiramatsu, M.; Hoson, A.; Niihara, K.; Langmuir 1998, 14, 3160 .

15. Mourão, H. A. J. L.; Mendonça, V. R.; Malaguitti, A. R.; Ribeiro, C.; Quim. Nova 2009, 32, 2181.

16. In, S.; Orlov, A.; Garcia, F.; Tikhov, M.; Wright, D. S.; Lambert, R. M.; Chem. Commun. 2006, 40, 4236.

17. Han, C.; Li, Z.; Shen, J.; J. Hazard. Mater. 2009, 168, 215.

18. Sahoo, C.; Gupta, A. K.; Pal, A.; Desalination 2005, 181, 91.

19. Ko, S.; Benerjee, C. K.; Sankar, J.; Composites B: Eng. 2011, 42, 579.

20. Zhu, B.; Sui, Z.; Wang, S.; Chen, X.; Zhang, S.; Wu, S.; Huang, W.; Mater. Res. Bull. 2006, 41, 1097.

21. Liu, G.; Li, G.; Qiu, X.; Li, L.; J. Alloys Compd. 2009, 481, 492.

22. Wang, M.; Dao, G.; Hu, L.; J. Solid State Chem. 2005, 178, 1996.

23. Lee, C. K.; Wang, C. C.; Lyu, M. D.; Juang, L. C.; Liu, S. S.; Hung, S. H.; J. Colloid Interface Sci. 2007, 316, 562.

24. Chai, L. Y.; Wei, S. W.; Peng, B.; Li, Z. Y.; Trans. Nonf. Metals Soc. Chin. 2008, 18, 980.

25. Soares, E. T.; Lansarin, M. A.; Moro, C. C.; Braz. J. Chem. Eng. 2007, $24,29$. 